\title{
Qualitative study of cardiologists' perceptions of factors influencing clinical practice decisions
}

\author{
Veena Manja, ${ }_{1}^{1}$ Gordon Guyatt, ${ }^{2}$ John You, ${ }^{3}$ Sandra Monteiro, ${ }^{2}$ Susan Jack ${ }^{4}$
}

- Additional material is

published online only. To view please visit the journal online (http://dx.doi.org/10.1136/ heartjn-2018-314339).

'Department of Surgery, University of California Davis, Davis, California, USA

${ }^{2}$ Department of Health Research Methods, Evidence and Impact McMaster University, Hamilton, Ontario, Canada ${ }^{3}$ Department of Medicine, McMaster University, Hamilton, Ontario, Canada

${ }^{4}$ School of Nursing, McMaster University, Hamilton, Ontario, Canada

\section{Correspondence to}

Dr Veena Manja, Department of Surgery, University of California Davis, Sacramento, CA 95817, USA; vmanja@ucdavis.edu

Received 24 October 2018 Revised 9 December 2018 Accepted 10 December 2018 Published Online First 12 January 2019

\section{Linked}

- http://dx.doi.org/10.1136/ heartjnl-2018-314593

\section{Check for updates}

(C) Author(s) (or their employer(s)) 2019. No commercial re-use. See rights and permissions. Published by BMJ.

To cite: Manja V, Guyatt G, You J, et al. Heart

2019;105:749-754.

\section{ABSTRACT}

Background Healthcare costs are increasing in the USA and Canada and a substantial portion of health spending is devoted to services that do not improve health outcomes. Efforts to reduce waste by adopting evidencebased clinical practice guideline recommendations have had limited success. We sought insight into improving health system efficiency through understanding cardiologists' perceptions of factors that influence clinical decision-making.

Methods In this descriptive qualitative study, we conducted in-depth interviews with 18 American and 3 Canadian cardiologists. We used conventional content analysis including inductive and deductive approaches for data analysis and mapped findings to the ecological systems framework.

Results Physicians reported that major determinants of practice included interpersonal interactions with peers, patients and administrators; financial incentives and system factors. Patients' insurance status represented an important consideration for some cardiologists. Other major influences included time constraints, fear of litigation (less prominent in Canada), a sense that their obligation was never to miss any underlying pathology, and patient demands. The need to bring income into their health system influenced American cardiologists' practice; personal income implications influenced Canadian cardiologists' practice. Cardiologists reported that knowledge limitations and logistical challenges limit their ability to assist patients with cost considerations. All these considerations were more influential than guidelines; some cardiologists expressed a high level of scepticism regarding guidelines.

Conclusions Clinical decision-making by cardiologists is shaped by individual, interpersonal, organisational, environmental, financial and sociopolitical influences and only to a limited extent by guideline recommendations. Successful strategies to achieve efficient, evidence-based care will require addressing socioecological influences on decision-making.

\section{INTRODUCTION}

A substantial portion of healthcare spending is for services that do not improve health outcomes, ${ }^{1}$ while interventions proven to improve health outcomes are underused. ${ }^{2}$ Given similar clinical information and circumstances, different physicians treating the same patient make very different decisions regarding testing and treatment. ${ }^{3}$ This type of variation in health services utilisation is common in cardiology in the USA ${ }^{4}$ and Canada,${ }^{5}$ and when there is a clear, optimal, evidence-based course of action, practice variation will be associated with disparities in quality and efficiency of care. ${ }^{6}$ Efforts to improve implementation of evidence-based strategies include clinical practice guidelines (CPG) intended to assist physicians in decision-making by appraising available research evidence and producing evidence-based recommendations for disease prevention, diagnosis and treatment. ${ }^{7}$ However, adoption of CPG recommendations by cardiologists is suboptimal. $^{8}$

Much of the literature addressing variation in care is based on statistical analyses of large databases. ${ }^{4}$ Studies seeking physician input have included surveys ${ }^{910}$ and focus groups ${ }^{1112}$ and have identified general challenges including physician perceptions and patient-related factors that result in evidence-practice gaps. A mixed-methods approach to explore context specific factors that influence practice decisions may provide a more nuanced understanding of clinical decision-making leading to evidence-practice gaps.

We therefore conducted a sequential explanatory mixed-methods study including two phases ${ }^{13}$ to identify and understand factors affecting cardiologists' decisions. In the first quantitative phase, a survey of 106 cardiologists evaluated extent of concordance with CPG recommendations and measured the rating of factors on decision-making ${ }^{14}$ and found that cardiologists rated the influence of evidence-based practice high in their decision-making even when they chose non-evidence-based, guideline-discordant management options. This qualitative phase seeks to understand the reasons why cardiologists chose guideline-discordant options when they report prioritising evidence-based care in their practices.

\section{METHODS}

\section{Study design}

The principles of qualitative description informed all methodological decisions related to sampling, data collection and analysis. Qualitative description $^{15}$ allows the researcher to stay close to the data, provide factual summaries of participants' experiences and perceptions, uses low inference in its description and is grounded in the principles of naturalistic inquiry.

\section{Sampling}

We chose a purposeful maximum variation sample of cardiologists who participated in the quantitative phase. Based on literature addressing optimal sample size in studies using qualitative interviews, ${ }^{16}$ 
our goal was to interview 12 to $21^{13}$ cardiologists in order to include diversity with respect to cardiologists' background and practice settings. ${ }^{17}$

\section{Data collection}

Cardiologists participated in face-to-face or telephone semistructured interviews conducted by the principle investigator (VM) between October 2017 and April 2018. Each interview lasted between 30 and $60 \mathrm{~min}$ and was digitally recorded. The results of the survey ${ }^{14}$ informed an initial draft of the interview guide by VM that was revised based on input of the authors and further refined to improve clarity and flow of questions based on experience using it in initial interviews. Online supplementary table 1 presents the final version of the interview guide.

\section{Data analysis}

The principles of conventional content analysis ${ }^{18}$ guided the analytic process. We audio-recorded the interviews, transcribed them verbatim, removed identifying information, and stored data in a password-protected computer. Data management and coding was performed by VM using Microsoft Excel and Microsoft Word. VM read the interview transcripts in their entirety several times to get familiar with the data. The senior author with extensive experience in qualitative research coded three of the interviews, reviewed samples of coded interviews and supervised the analytic process. The results of ongoing analysis were reviewed with the authors during regular meetings (approximately monthly) and disagreements were resolved by discussion and consensus. Using a broad-based coding process to create a collection of experiences and accounts, we regrouped the data according to themes. Initially a primarily inductive approach led to development of open codes based on the concepts within the text which were collapsed into categories followed by identification of major themes. Analysis of the interviews revealed a pattern of interconnected individual, interpersonal and organisational factors that influenced decision-making aligning with the ecological systems theory that specifies nested environmental systems; results were mapped to the social-ecological systems theory framework. ${ }^{19}{ }^{20}$ Although originally developed to explain child development, investigators have used this theory in studies assessing a variety of social influences on decision-making. ${ }^{2021}$ This process provided an opportunity for theoretical triangulation.

\section{RESULTS}

Of the 25 cardiologists approached, 21 agreed to participate; 12 interviews occurred face-to-face and 9 by telephone. Participating cardiologists' (USA =18, Canada $=3$ ) demographic characteristics are presented in table 1 .

\begin{tabular}{lc} 
Table 1 Demographic characteristics of participating cardiologists \\
\hline \multicolumn{2}{l}{ Number } \\
\hline Practice-setting \\
$\quad$ Academic \\
$\quad$ Private \\
Sex \\
$\quad$ Male \\
$\quad$ Female \\
Years in practice \\
$\quad<20$ \\
$>20$ & 5 \\
\hline
\end{tabular}

\section{Overview of results}

Cardiologists explained that in an ideal world, they would make decisions based on a combination of their knowledge of the pathophysiology of disease, evidence, CPG recommendations, prior personal clinical experiences and patients' values and preferences. However, in the real world, practice restrictions and community norms were identified as important and their decisions were sometimes influenced by what care the patient could afford. Cardiologists balanced the scientific evidence with patient and peer expectations and organisational, environmental and societal norms and constraints. Figure 1 depicts the various factors and their inter-related nature that influence decision-making. Online supplementary table 2 presents results of the analysis mapped to the socioecological framework. ${ }^{19} \mathrm{~A}$ brief description of interactions between the different domains and systems with illustrative quotes is presented. Additional quotes supporting the themes and categories are presented in online supplementary table 3 .

\section{Practice expectations and patient preferences}

Cardiologists described experiencing conflicts of interest that influence decision-making. In addition to the explicit conflict with fee-for-service reimbursement, ordering tests and procedures influences clinical productivity, bonuses and maintenance of procedure volumes. An academic cardiologists stating: 'There is definitely a need to fill the cath [cardiac-catheterization] schedule, fill the time, do those procedures, looking like we are doing procedures, so there is a pressure to do that sometimes'. Cardiologists noted pressure from managers and service chiefs in private and academic practice to increase tests and revenue; stating, for instance 'When we tried to limit echo referrals, we actually got word from our chief, 'don't do that, echo is one of the major income generating sections of cardiology'. Patients perceive testing as representative of good healthcare, as noted by a cardiologist in private practice, 'The patients feel everything has been checked and everything is fine'. A cardiologist mentioned that if the patient's symptoms did not meet insurance pre-authorisation criteria, he sometimes modifies his note:

You end up kind of stretching the truth, or composing your history such that the symptoms sound maybe more concerning than maybe you think they are, just so he qualifies for getting a stress test with nuclear imaging.

\section{Peer expectations and appropriate-use-criteria}

There was general agreement among cardiologists that the appropriate-use-criteria as a tool to limit unnecessary testing are not very useful in clinical practice because of the broad categorisations, lack of evidentiary support for the appropriate-use-criteria recommendations and lack of 'buy-in' by practicing cardiologists. Respondents identified peer expectations, need to collaborate and community norms as strong drivers of clinical decisions, often overriding the appropriate-use-criteria. One cardiologist noted, 'The primary care provider sends the patients to you for certain things, there is an expectation [of tests] and they look for the results'. Another commenting on the influence of practice norms and expectations noted:

I try to follow appropriate-use-criteria, and I have been criticized for being too conservative and not sending enough people to the [catheterization] lab. And sometimes it is difficult to ignore the peer pressure which may then drive decisions, especially for junior faculty. And the (more) junior they are the more likely they are to be, in my estimate, more aggressive than indicated by the criteria or by the patient. 


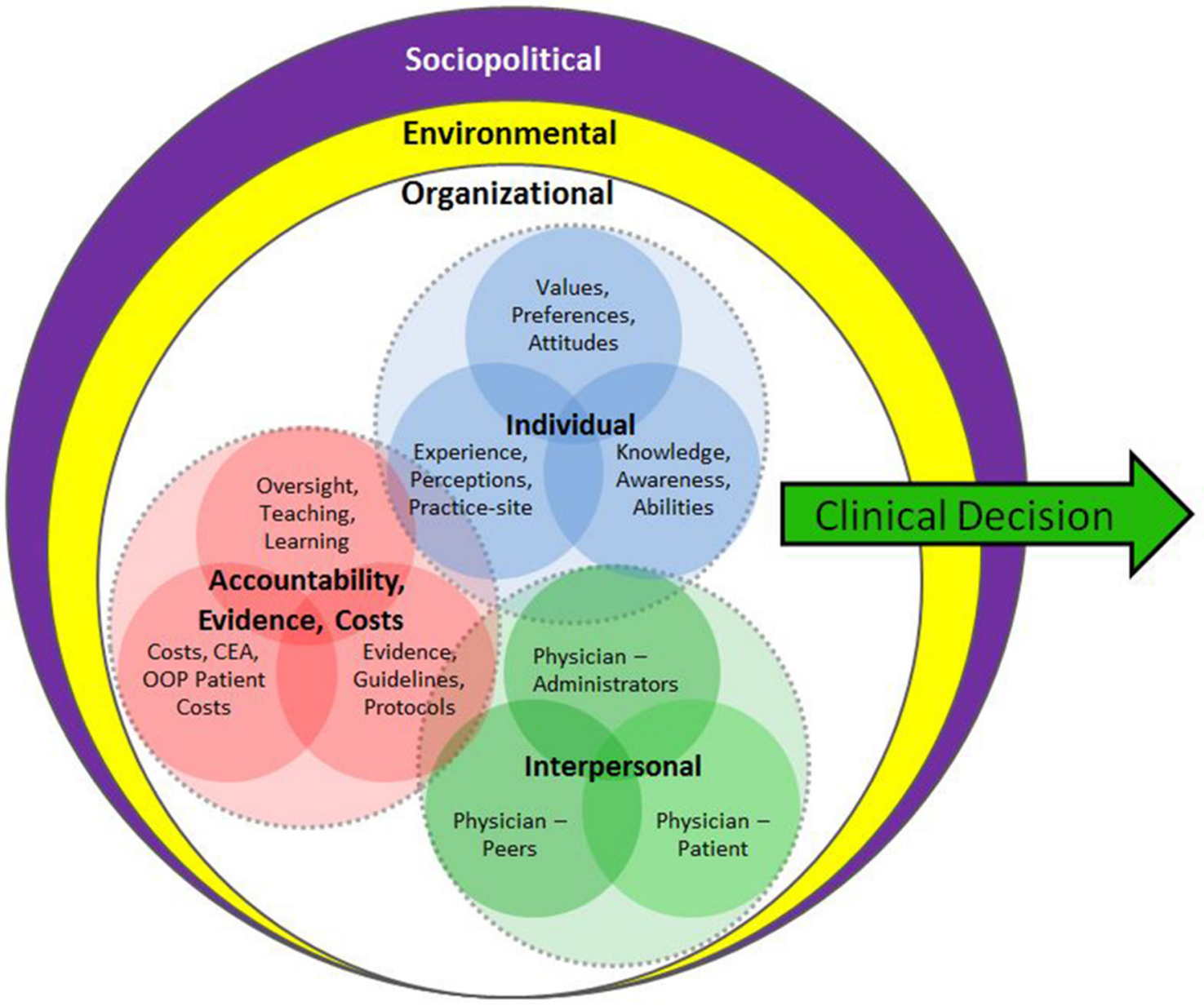

Figure 1 Domains/Factors influencing clinical decision-making. CEA,cost effectiveness analysis; OOP, out of pocket.

\section{Handling uncertainty, medicolegal concerns and scepticism regarding CPG recommendations}

Cardiologists varied in their level of comfort with uncertainty; some preferred limited testing noting, 'Often patients will accept that [reassurance] and you don't need to look further'. However, most were driven by a need for certainty, especially in a litigious environment. A common rationale used was the perception that cardiologists need to perform more tests because they are dealing with life and death situations. Cardiologists often quoted CPG recommendations as justification for their clinical decisions when they aligned with their practice, but found them lacking when their practice diverged from CPG recommendations. One common response was that CPGs are written by researchers out of touch with the needs of busy clinical practice, summarised thus by a cardiologist:

Guidelines are often developed by academic guys who are more purists in terms of the data and do not see many patients, there is a disconnect between the purely academic people and those who have the responsibility to treat people. I think when you have the responsibility not to miss anything - that is where you get the divergence from the guidelines.

Another concern with the guidelines was that they were unwieldy, one cardiologist noting, All the guidelines are so voluminous that they become almost irrelevant. They try to dig into the minutia so much that they lose the forest for the trees'.

\section{Cost considerations}

When asked about the influence of cost on their decision-making, cardiologists acknowledged ignorance about the costs of testing; the charges to the patient and some noted an inability to acquire information on costs. One academic cardiologist stated:

I have a patient who wants to pay cash for a test, how much does it cost? My own organisation will beat around the bush; say, 'we can't tell you, it all depends,' blah blah.... This is ridiculous. I mean the rest of the world it is very clear, I hire a plumber, he says it is $\$ 50$ an hour or it's a weekend it is $\$ 67$ an hour. Okay I know that, then why in medicine we've made this a game of cat and mouse, I have no idea. Why should a CT scan cost be different based on your insurance, based on your ZIP Code? This is the conspiracy theory of medicine!

Respondents perceived an urgent need to consider costs at a societal level, but also expressed a reluctance to consider cost when making clinical decisions for an individual patient. One academic cardiologist commented, 'I think as a society we need to consider cost-effectiveness because we just cannot afford to keep paying for every latest and greatest therapy that has some marginal benefit'. However, he went on to say:

I think that the way I take care of the patient is not necessarily the way I want society to take care of everybody. I am my patient's advocate and if I think they should have something that even though is not the standard of care, I feel obligated to do that. 
Others modified the treatment plan based on the patient's insurance and ability to pay; one cardiologist commented on her colleague's practice of prescribing less effective cheaper options to patients with limited coverage:

\begin{abstract}
She puts the patient on amiodarone [instead of a defibrillator], she sits down and talks to them so the patient is aware of the trade-offs. It just kills me to decide to treat different patients differently based on their insurance and affordability.
\end{abstract}

Some cardiologists highlighted a recent development related to cost: to improve efficiency and encourage cost-consciousness among physicians, hospitals and insurance companies collect and share physician-level cost data sometimes used in reimbursement and referral decisions. One cardiologist noting:

\begin{abstract}
So, there is a financial incentive to do these tests from an individual practitioner standpoint. The flip side of that, it is interesting how this is evolving, the referring physicians are often graded on their cost of care for their patients, so when they refer somebody to a cardiologist who is going to do all these fancy tests versus one who only orders them when necessary, there is a different cost of care and they are going to favour the one who is not running around doing tests. I do think there have to be some economic drivers to force people to do the right thing.
\end{abstract}

\section{Canadian context}

Despite fundamental differences in healthcare financing between Canada and USA, many similarities were noted in practice patterns by cardiologists in the two systems. Canadians were influenced by patient expectations, financial incentives (in private-practice) and preference for testing to decrease uncertainty. Perceived differences were due to differences in funding mechanisms and medicolegal concerns (less pressing in the Canada) leading to a perception of decreased need for practicing 'defensive-medicine'.
In spite of CPG recommendation against its use in specific contexts, cardiologists noted several perceived benefits from an echocardiogram. The cited reasons provide insights into overuse of medical technologies and are summarised in figure 2 . Mapping the differences in rating of influential factors in disposition decisions for a patient with non-cardiac chest pain in our case-based survey, ${ }^{14}$ figure 3 illustrates varying contextual influences leading to different clinical decisions. Ratings were significantly different $(\mathrm{p}<0.0001)$ between cardiologists who chose to discharge the patient compared with those who chose to admit the patient or to perform more tests. Portraying the results in a visual format may enhance appreciation of contextual influences and assist with developing strategies to implement high-value care.

\section{DISCUSSION}

Our results illustrate the complexity and multiple factors that influence clinical decision-making by cardiologists in the USA and Canada. Physician behaviour leading to unnecessary testing is driven by the need to fulfil practice expectations (productivity, costs and procedure volumes), time constraints, financial incentives, medicolegal concerns, peer-pressure and patient expectations. Cardiologists do consider evidence of effectiveness and CPG recommendations in decision-making, but in many instances they play a secondary role. When cardiologists diverge from CPG recommendations, it is generally not due to lack of knowledge but because of competing influences. Although American patients are increasingly requesting information on costs, the inability to assist with cost information was a source of frustration for cardiologists who expressed an urgent need to improve cost transparency in healthcare.

American cardiologists, but not their Canadian counterparts, noted the practice of gathering and reporting physician specific cost data to provide feedback to individual physicians

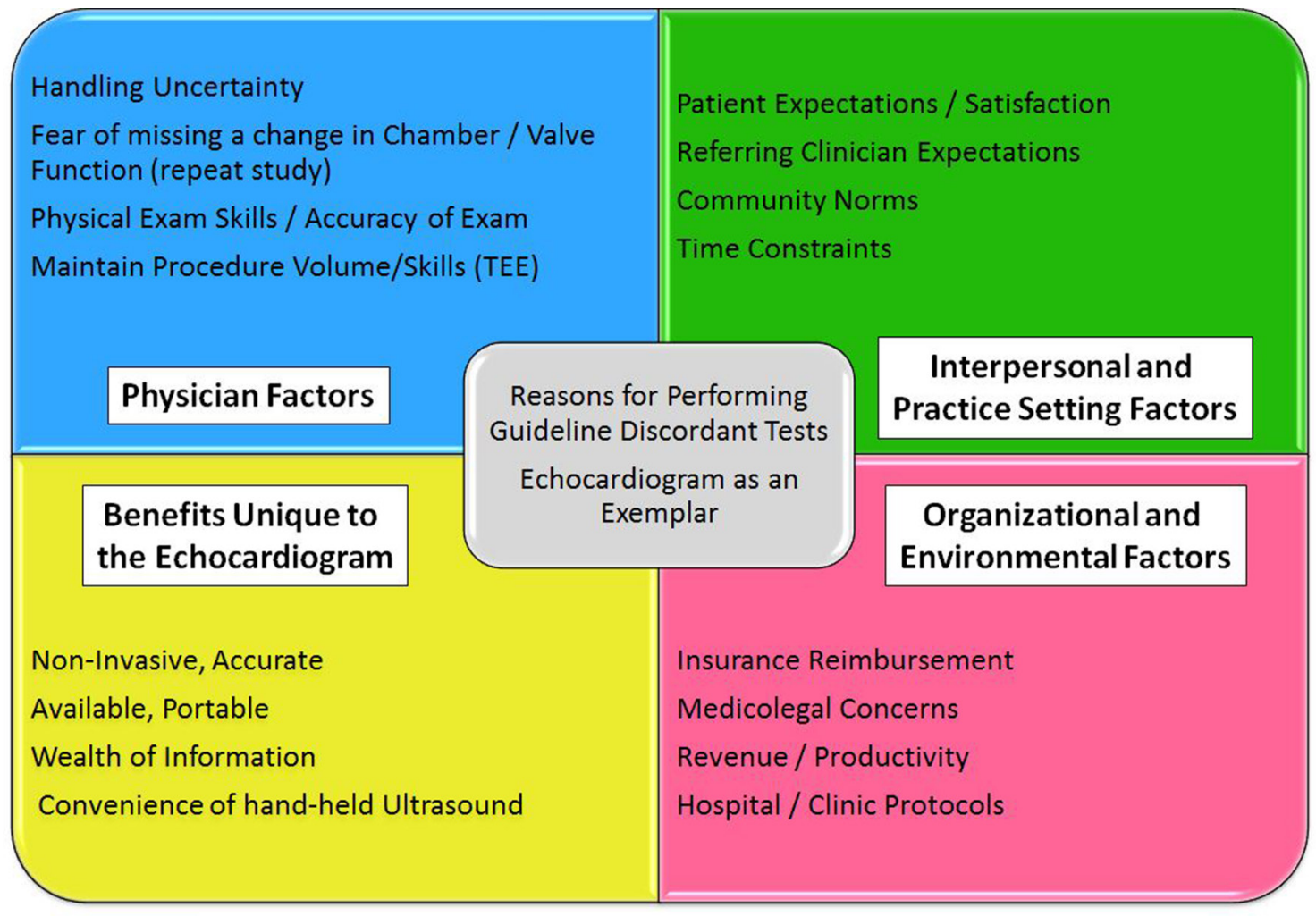

Figure 2 Insights into factors that promote guideline discordant care. TEE, transesophageal echocardiogram. 

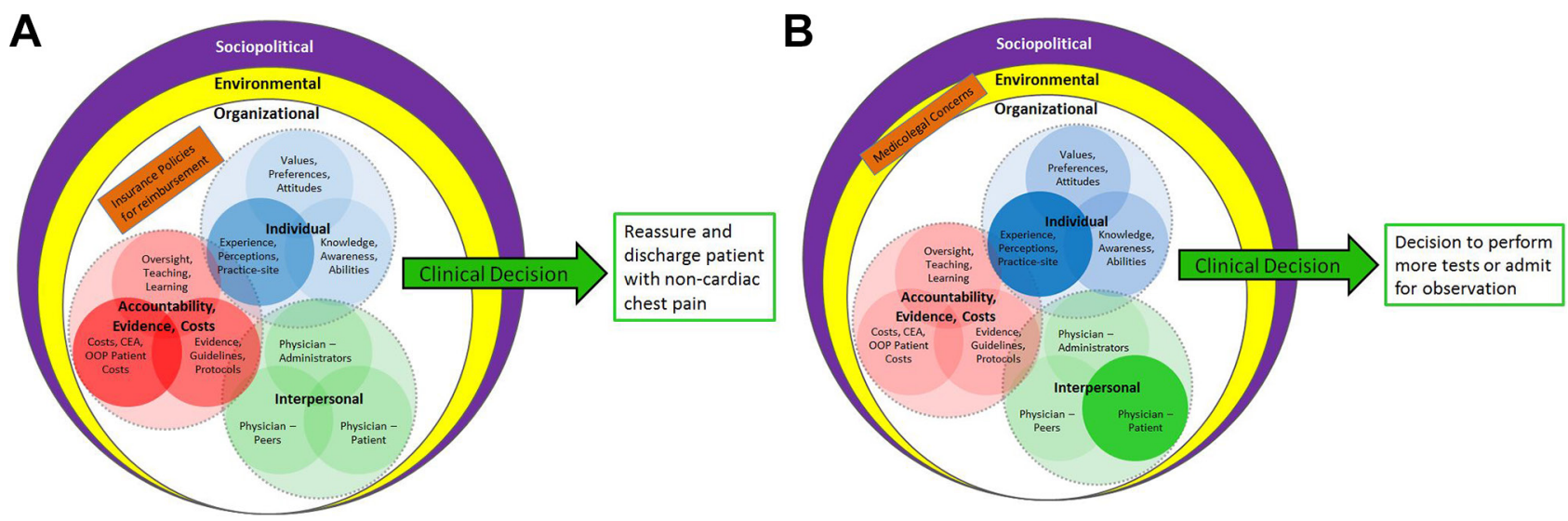

Figure 3 (A) Factors influencing decision to discharge a patient with non-cardiac chest pain. (B) Factors influencing decision to perform further testing or admit for observation in a patient with non-cardiac chest pain.

by hospitals and third-party payers in an effort to encourage cost-consciousness. Respondents noted this practice to be an increasing and evolving influence on decision-making with potentially important impact on clinical practice. They perceived themselves as caught between competing pressures to do more and initiatives to do less.

Strengths of our study include its use of the prior quantitative study to inform design ${ }^{14}$ and varying perspectives of the team in creating and modifying the survey. Recruitment of cardiologists practicing in diverse settings and backgrounds enhance the transferability of our findings. Duplicate review of a set of interview transcripts ensured identification and labelling of key constructs. The application of strategies to address credibility including methodological coherence, investigator credibility and triangulation further enhance the overall rigour and trustworthiness of the data. Mapping our results to the socioecological systems theory framework provides a structure for considering the causes of unwarranted variation and for promoting potential solutions.

Limitations include participation by few women cardiologists. There may be gender differences in handling uncertainty and negotiating treatment plans with patients leading to adoption of different management strategies. Interviews with more Canadian cardiologists, especially in the private practice setting, would have added to the understanding of similarities and differences between the two settings. Although mapping our findings to the ecological systems theory framework provided theoretical triangulation, this framework was not developed specifically for studying decision-making. Validating our results will require replication in future studies. Due to time constraints, we did not pursue member checking; confirmation of findings by interview participants may have enhanced our results.

Our findings are consistent with other reports. In a qualitative study addressing the determinants of appropriate use of echocardiography, Fonseca and colleagues ${ }^{22}$ found that physician factors including training, experience, handling uncertainty and perception of peer and patient expectations influenced echocardiogram use. Similarly, Hisham and colleagues ${ }^{23}$ found that despite having positive attitudes about evidence and CPGs, doctors' practice was often not evidence-based due to barriers including heavy workload and workplace culture. Additionally, studies in the literature suggest that physicians' perceptions of benefits and harms of tests and treatment ${ }^{24}$ may be inaccurate leading to 'therapeutic-illusion' and a tendency to 'err on the side of caution'.
Our results provide credence to critiques of the industrialisation and profit focus of American healthcare. ${ }^{25}$ Financial incentives and the pressure to meet productivity goals drive overutilisation of unnecessary testing and treatment, limit time with the patient and move the focus towards technical testing. This phenomenon is not limited to cardiology; evidence for variation in care attributable to differences in reimbursement mechanisms and supplier-induced demand exist for many specialties within healthcare including surgery, ${ }^{26}$ nephrology, ${ }^{27}$ neonatology ${ }^{28}$ and obstetrics. ${ }^{29}$ Published studies in diverse health settings in countries aside from the USA ${ }^{28} 30$ suggest that financial incentives lead to similar practice patterns by physicians in diverse settings and have a central role in efforts to change practice.

Our results make evident the unintended consequences of current regulations, practices and policy. Peer, patient and community expectations play a major role in guideline-divergent decision-making. This finding may explain the limited success of approaches to increase the value of care by focusing on strategies to improve physician's knowledge.

Our findings have practice, research and policy implications for addressing medical waste. As long as financial and productivity incentives persist they will continue to drive overuse. Fundamental reorganisation of practice incentives is essential to bring about substantial change in decision-making. The influence of the interaction of the contextual factors identified in this study within the current complex healthcare environment needs further research. Increasing use of qualitative studies including focused ethnography may be helpful in this regard. Methods to encourage patient-centred, efficient and evidence-based care need further study before implementation.

\section{CONCLUSION}

Clinical decision-making by cardiologists is shaped by individual, interpersonal, organisational, environmental and sociopolitical influences and to a limited extent by CPG recommendations. Our results suggest that the marginal success of current initiatives to optimise efficiency and value of healthcare results from their ignoring the most powerful factors that influence clinical decisions. To develop successful interventions that result in positive, fundamental changes in practice will require a sophisticated understanding of the drivers of overuse in the social and ecological context. Changes in reimbursement structure and greater transparency in healthcare financing are essential to achieving efficient, evidence-based patient-centred care. 


\section{Key messages}

\section{What is already known on this subject?}

- A substantial portion of health spending is devoted to services that do not improve health outcomes while services known to improve outcomes are underused. Efforts to improve quality and efficiency of healthcare have had limited success.

\section{What might this study add?}

- Individual physician-related factors as well as interpersonal, organisational and environmental factors play an important role in clinical decision-making. In daily clinical practice, while evidence-based guideline recommendations are considered important by physicians, they play a secondary role in decision-making because of competing influences. Ignoring the most powerful factors that influence clinical decisions is likely responsible for the marginal success of current initiatives to optimise efficiency and value of healthcare.

\section{How might this impact on clinical practice?}

- Strategies to improve implementation of high-value care must consider these contextual factors to be successful in their efforts. Fundamental reorganisation to practice incentives is essential to bring about meaningful change in decision-making.

Acknowledgements The authors gratefully acknowledge the interview participants for their time and input. They would also like to acknowledge the comments and suggestions of the anonymous reviewers of this paper which greatly improved the final manuscript.

Contributors VM contributed to the conception, and all authors to the planning and design of the work. VM contributed to acquisition, VM and SJ to the analysis and all authors to the interpretation of data. VM drafted the initial draft of the manuscript which was revised based on extensive input from all authors. All authors give final approval of the version published. VM is responsible for the overall content as guarantor.

Funding The authors have not declared a specific grant for this research from any funding agency in the public, commercial or not-for-profit sectors.

Competing interests None declared.

Patient consent Not required.

Ethics approval The Institutional Review Board at the University at Buffalo, Buffalo, New York, USA and the Hamilton Integrated Research Ethics Board at McMaster University, Hamilton, Ontario, Canada.

Provenance and peer review Not commissioned; externally peer reviewed.

\section{REFERENCES}

1 Schwartz AL, Landon BE, Elshaug AG, et al. Measuring low-value care in medicare. JAMA Intern Med 2014:174:1067-76.

2 Levine DM, Linder JA, Landon BE. The quality of outpatient care delivered to adults in the United States, 2002 to 2013. JAMA Intern Med 2016:176:1778-90.

3 Mercuri M, Natarajan MK, Norman G, et al. An even smaller area variation: differing practice patterns among interventional cardiologists within a single high volume tertiary cardiac centre. Health Policy 2012;104:179-85.
4 Kumar A, Fonarow GC, Eagle KA, et al. Regional and practice variation in adherence to guideline recommendations for secondary and primary prevention among outpatients with atherothrombosis or risk factors in the United States: a report from the REACH registry. Crit Pathw Cardiol 2009;8:104-11.

5 Wijeysundera DN, Austin PC, Beattie WS, et al. Variation in the practice of preoperative medical consultation for major elective noncardiac surgery: a population-based study. Anesthesiology 2012;116:25-34.

6 Krumholz HM. Variations in health care, patient preferences, and high-quality decision making. JAMA 2013;310:151-2.

7 Woolf SH, Grol R, Hutchinson A, et al. Clinical guidelines: potential benefits, limitations, and harms of clinical guidelines. BMJ 1999;318:527-30.

8 Greene SJ, Butler J, Albert NM, et al. Medical therapy for heart failure with reduced ejection fraction. J Am Coll Cardiol 2018;72:351-66.

9 Zikmund-Fisher BJ, Kullgren JT, Fagerlin A, et al. Perceived Barriers to Implementing Individual Choosing Wisely ${ }^{\circledR}$ Recommendations in Two National Surveys of Primary Care Providers. J Gen Intern Med 2017;32:210-7.

10 Kanzaria HK, Hoffman JR, Probst MA, et al. Emergency physician perceptions of medically unnecessary advanced diagnostic imaging. Acad Emerg Med 2015;22:390-8.

11 Bishop TF, Cea M, Miranda Y, et al. Academic physicians' views on low-value services and the choosing wisely campaign: A qualitative study. Healthc 2017;5(1-2):17-22.

12 Sabbatini AK, Tilburt JC, Campbell EG, et al. Controlling health costs: physician responses to patient expectations for medical care. J Gen Intern Med 2014;29:1234-41.

13 Manja V, Monterio S, Guyatt G, et al. Understanding the factors that influence clinical decision-making - a sequential explanatory mixed methods study protocol. Eur J Pers Cent Healthc 2018;6:329-328.

14 Manja V, Guyatt G, Monteiro S, et al. Sources of practice variations in cardiology - The influence of clinical context, cost, physicians' perceptions and practice considerations. Eur J Pers Cent Healthc 2018;6:492

15 Neergaard MA, Olesen F, Andersen RS, et al. Qualitative description - the poor cousin of health research? BMC Med Res Methodo/ 2009;9:52.

16 Guest G, Bunce A, Johnson L. How many interviews are enough? An experiment with data saturation and variability. Field Method 2006;18:59-82.

17 Nelson J. Using conceptual depth criteria: addressing the challenge of reaching saturation in qualitative research. Qualitative Research 2017;17:554-70.

18 Hsieh HF, Shannon SE. Three approaches to qualitative content analysis. Qual Health Res 2005;15:1277-88.

19 Bronfenbrenner U. Toward an experimental ecology of human development. Am Psychol 1977;32:513-31.

20 Hickey N, Harrison L, Sumsion J. Using a socioecological framework to understand the career choices of single- and double-degree nursing students and double-degree graduates. ISRN Nurs 2012:2012:1-10.

21 Qiao S, Li X, Zhou Y, et al. Factors influencing the decision-making of parental HIV disclosure: a socio-ecological approach. AIDS 2015;29(1):S25-34.

22 Fonseca R, Jose K, Marwick TH. Understanding decision-making in cardiac imaging: determinants of appropriate use. Eur Heart J Cardiovasc Imaging 2018;19:262-8.

23 Hisham $\mathrm{R}, \mathrm{Ng} \mathrm{CJ}$, Liew SM, et al. Why is there variation in the practice of evidencebased medicine in primary care? A qualitative study. BMJ Open 2016;6:e010565.

24 Hoffmann TC, Del Mar C. Clinicians' expectations of the benefits and harms of treatments, screening, and tests: a systematic review. JAMA Intern Med 2017;177:407-19.

25 Montori V. Why we revolt: a patient revolution for careful and kind care. Patient Revolution 2017

26 Nguyen LL, Smith AD, Scully RE, et al. Provider-induced demand in the treatment of carotid artery stenosis: variation in treatment decisions between private sector fee-forservice vs salary-based military physicians. JAMA Surg 2017;152:565-72.

27 Kurella Tamura M, Thomas IC, Montez-Rath ME, et al. Dialysis initiation and mortality among older veterans with kidney failure treated in medicare vs the department of veterans affairs. JAMA Intern Med 2018;178:657-64.

28 Shigeoka H, Fushimi K. Supplier-induced demand for newborn treatment: evidence from Japan. J Health Econ 2014;35:162-78.

29 Grant D. Physician financial incentives and cesarean delivery: new conclusions from the healthcare cost and utilization project. J Health Econ 2009;28:244-50.

30 Jürges $H$, Köberlein J. What explains DRG upcoding in neonatology? The roles of financial incentives and infant health. J Health Econ 2015;43:13-26. 